by the Special Hospitals' Services Authority in relation to trial leave to another hospital. It is usual practice that patients from special hospitals are sent to another hospital on six months trial leave prior to their formal transfer but when it comes to authorising a formal transfer different sets of rules seem to apply. Our observations are based on a number of patients transferred to a medium secure unit.

With regard to restricted patients, the Secretary of State does not appear satisfied with a good progress report over six months' duration and takes an overcautious view advising the Special Hospital Authority to extend the trial leave period, even when the consultants from the special hospital and the receiving hospital have agreed and supported the transfer of the patient. Such uncertainty over formal transfer and extension of trial leave can have detrimental effects on patients' progress.

Regarding trial leaves of unrestricted patients, the Special Hospitals' Services Authority refuses to grant an extension to trial leave, even when there are good grounds and both consultants support such an extension. Such a policy forces the receiving hospital to decide whether to accept a formal transfer after six months or to return the patient to the special hospital. Both of these options, if taken hastily, may not be in the best interest of the patient and can lead to inappropriate placements.

We were faced with a similar dilemma at the end of a trial leave period in respect of two patients transferred from a special hospital. Miss $A$, detained under section 37 and 41 of the Mental Health Act, behaved well and progressed satisfactorily and could not understand why she was not formally transferred while $\mathrm{Mr} \mathrm{B}$, detained under section 37, was transferred formally after six months despite displaying aggressive and violent behaviour.

We should be glad to hear the experiences of other psychiatrists and the views of colleagues in special hospitals and the Special Hospitals' Services Authority.

*ANIl Kumar, Medium Secure Unit, Calderstones NHS Trust, Whalley, Clitheroe BB7 9PE and MEENA AGARWAL, 46 Larkhill Cottages, Old Langho, Blackburn, BB6 8AR

*Correspondence

\section{Conceptualisation of depression}

Sir: Jadhav \& Littlewood refer to the study by Weiss et al, (1992) as evidence that "inculcating professional medical models do not necessarily bring about reduced stigmatisation or improved compliance" (Psychiatric Bulletin, September 1994, 18, 572-574). I beg to differ. Patients in that study showed an increased medication ad- herence rate $(79 \%$ for study patients as opposed to $46 \%$ for non-study patients, $P<0.0001$, Fisher's exact test) after being given a 'medical model' of leprosy during the initial interview. That paper did not report any findings on stigma at follow-up.

However, Jadhav \& Littlewood raise important questions which remain unanswered. Is a 'biomedical model' the only way to conceptualise depression? The Defeat Depression campaign, in title and content (Paykel \& Priest, 1992), carries an implicit message that depression is a 'disease' one 'catches' and which can be 'understood' and 'treated' with no reference to the socio-cultural context. It emphasises the primacy of the biomedical model when there is no evidence that a belief in or practice of such a model is associated with better outcome or greater patient acceptance.

PAYKel, E.S. \& PRIEST, R.G. (1992) Recognition and management of depression in general practice: consensus statement. British Medical Journal, 305, 1198-1202.

Weiss, M., Doongan, D. et al, (1992) The explanatory model interview catalogue (EMIC): a contribution to crosscultural research methods from a study of leprosy and mental health. British Journal of Psychiatry. 160, 819830.

SOUMITRA PATHARE, Academic Unit of Psychiatry, St Thomas' Hospital, London SE1

\section{Sexist language}

Sir: I agree with Dr Y. K. Mirza (Psychtatric Bulletin, September 1994, 18, 584) that the use of the word "Sir" in the correspondence section may be construed as sexist. As Dr Mirza invites us to suggest a single non-sexist word, I would propose the word "Editor" be used instead.

TALAL Al RuBAIE, Psychotherapist in private practice, 41 Denison Close, East Finchley, London N2 aJU

Sir: Dr Mirza is rather lacking in imagination if he cannot think of an alternative to 'Sir' for prefacing a letter to the Editor of the Bulletin. (Psychiatric Bulletin, September 1994, 18, 584). What about 'Dear Dr Kerr' as I have started this missive (if it appears in the pages of the Bulletin prefaced 'Sir', that is not how it left my desk) or Dear Editor, or even Dear Colleague? It is possible to start a letter with a salutation of more than one word.

I found the thinly velled and unsympathetic sarcasm of both Dr Mirza's and Dr Steinberg's letters quite offensive. This kind of attitude to the issue of sexism is a much greater problem than the consecutive occurrence of the letters M.A.N. in a word.

LiNDA R. MonTAGUE, Trafford General Hospital, Manchester M41 5SL 